- Optimization of imaging resources by surgical team from $33 \%$ to $17 \%$

- $88 \%$ of patients with abdominal pain had a medical diagnosis at discharge.

Conclusion

- Abdominal pain is a common presentation in the paediatric population, mostly benign and self-limiting.

- Abdominal pain should be assessed by general paediatricians first and then referred to surgical colleagues if deemed appropriate to avoid unnecessary investigations and imaging.

\section{P45 REVIEW OF DIAGNOSIS AND MANAGEMENT OF COELIAC DISEASE IN A DISTRICT GENERAL HOSPITAL IN THE NORTH WEST}

Ban Alkaaby, Zahra Khan, Suparna Dasgupta. Macclesfield General Hospital

10.1136/flgastro-2021-bspghan.54

Background The guidance for Coeliac Disease (CD) has evolved over the last few years. The highlight of the ESPGHAN guidelines from January 2020 is that high serological markers (tTG and EMA) are now the requirements for confirmation of $\mathrm{CD}$ regardless of symptoms.

Aims

1. To review management of patients and compare it with the latest ESPGHAN/BSPGHAN guidelines.

2. To review time to confirmed diagnosis from the start of symptoms.

3. To look at provision of Gluten free products at schools and Primary Care service.

Methods Retrospective data collected from Coeliac database from January 2018 till November 2020 in a District General Hospital with provision for a dedicated Coeliac Clinic supported by a Specialist Dietician. We reviewed the presentation of symptoms, methods of confirmation of CD, time duration from onset of symptoms till diagnosis, provision of gluten free meals at schools and availability of gluten free products on prescription.

Results 35 patients were diagnosed with CD, 31 were symptomatic and 4 were asymptomatic.

$27 / 31$ of the symptomatic patients had high level tTG (>10 times the upper limit), 20 had positive EMA and HLA DQ2/DQ8 for positive confirmation. The two negative EMA were referred for biopsy.

Three had HLA typing but had high tTG on 2 separate occasions which confirms diagnosis. 2 had only one high tTG and HLA but no EMA or second high tTG so they did not meet the diagnostic criteria.

4 symptomatic patients with low tTG $<10 \mathrm{x}$, had biopsy confirmation. 4 asymptomatic patients had high tTG and +ve EMA.

Of 28 patients referred through Primary Care, 7 were screened and referred with symptom duration of circa 4 to 12 months, another 7 were diagnosed through screening due to positive family history or type 1 Diabetes Mellitus and 14 cases had no specific duration of symptoms recorded. Remaining seven were diagnosed by hospital paediatricians due to different presentations.

Most parents stated that they were providing packed lunch box even if the school provided some gluten free meals as the menu choice lacked variety. Patients with Type 1 diabetes found a packed lunch easier for carbohydrate counting. Parents felt tailor made menu recommendation from the dietician to the school would be beneficial for families and the school.

Conclusion and Recommendation We thus identified that $82 \%$ $(n=29)$ of patient had met the criteria for diagnosis of CD as per 2015 guidelines, but if 2020 guidelines were applied then $94.2 \%(n=31)$ would have meet the criteria.

Recommendations

1. Identifying duration of time needed for the child to be screened will help to raise awareness within primary care practice. This will be audited in the future.

2. There is a large knowledge gap in schools about CD and the importance of convenient access to gluten free meals in enhancing compliance with gluten free food in children. Offering tailor made presentations to the local schools will address this issue. A further review to identify if similar knowledge gap exists in schools regionally is planned.

\section{P46 REVIEW THE DIAGNOSIS OF IBD IN CHILDREN WITH AILD (AUTO IMMUNE LIVER DISEASE) -8 YEARS' EXPERIENCE IN A TERTIARY CENTRE}

Maria Misiou, Ben Hope, Huey Miin Lee, Marianne Samyn, Babu Vadamalayan. King's College Hospital, London

\subsection{6/flgastro-2021-bspghan.55}

Background The association of autoimmune liver disease (AILD) and inflammatory bowel disease (IBD) is well documented. IBD affects about $45 \%$ of children with autoimmune sclerosing cholangitis (AISC) and about 20\% of those with autoimmune hepatitis (AIH). The aim of this study was to describe the clinical features of AILD associated with IBD and to evaluate the role of fecal calprotectin and the time to look for IBD in patients with primary diagnosis of AILD.

Methods We have conducted a retrospective review of paediatric patients with primary diagnosis of AILD and IBD between 2010 and 2018. Patients who were referred or diagnosed with IBD first were excluded. Diagnosis of IBD was based on clinical history, endoscopic appearance and histology findings. AILD patients were classified to AIH or AISC according to histology, radiology results and circulating antibodies. Patients' demographics, symptoms, FC, blood tests, timing before IBD diagnosis and treatment were collected.

Results 114 patients with AILD were identified: 74 (64\%) had screening with FC, 48/74 (64\%) had abnormal and 26 (35\%) normal test. $67 \%$ of FC performed at the diagnosis of liver disease. 48 patients had at least one endoscopy, 25 $(52 \%)$ were diagnosed with IBD, 8 patients had normal endoscopy but positive FC of mean value 162, (range 60332). 15 had no endoscopy despite abnormal FC. 6/26 patients with normal FC underwent endoscopy which was normal. Reason was persistently elevated liver enzymes, relapse of AILD or ongoing bowel symptoms. 25/114 (21\%) patients were diagnosed with IBD, 14/25 (56\%) had AISC, the rest $\mathrm{AIH}$. 25\% had family history of autoimmunity. Mean FC at diagnosis of IBD was 646 (range 60-4004). 14 were males and the mean age at diagnosis was 10 years. 18/ 25 (78\%) had ulcerative colitis (UC), 2/25 (0.8\%) Crohn's disease and $5 / 25(2 \%)$ indeterminate colitis(IBDU). 12/14 
patients with AISC had UC and 2 IBDU. 40\% of patients had simultaneous diagnosis of AILD and IBD, all presented with symptoms of bowel disease and abnormal GGT and/or aminotransferase activity (6/10 had PR bleeding). 15 (60\%) patients were diagnosed with AILD and concomitant IBD after 19 months (mean time). In 7/15 gut symptoms improved since immunomodulators started but FC was raised, $3 / 15$ had no gut symptoms but raised FC on screening and $5 / 15$ developed bowel symptoms after liver diagnosis, in 3 of them FC was raised since liver diagnosis. Endoscopic features included pancolitis in $60 \%$ and ileitis in 20\%. Histological features were more consistent with those of mild to moderate UC (78\%). All patients diagnosed with AILD were started on steroids, 9/25 were already on Azathioprine before the endoscopic assessment for IBD.

Conclusions $20 \%$ of patients with primary diagnosis of AILD had IBD. 40\% had simultaneous diagnosis; all had raised FC since AILD was identified. We recommend FC routinely in children with AILD for the early diagnosis of IBD. Colonoscopy should be considered in patients with symptoms of IBD and the ones with clearly elevated FC. The timing of the assessment is of paramount as immunosuppresive treatment can mask symptoms and change the disease activity.

\section{P47 SETTING UP A REGIONAL HOME CALPROTECTIN SERVICE DURING THE COVID-19 PANDEMIC OFFERING HOSPITAL-BASED TESTING TO LOCAL AND REGIONAL PAEDIATRIC IBD PATIENTS IN WESSEX}

Claire Barnes, Chris Roberts, Jo Ward, Tracy Coelho, Akshay Batra, Robert Mark Beattie, Efrem Eren, Nadeem Afzal. University Hospital Southampton

\subsection{6/flgastro-2021-bspghan.56}

Background The first wave of the COVID-19 pandemic in the UK severely restricted our regional paediatric GI outpatient services affecting our ability to assess patients in hospital, further compounded by distance of travel of patients (An audit form 2019 showed $70 \%$ of patients endoscoped were from outside the local, rather than Southampton area). The issue was further compounded by some DGH's, who stopped offering the calprotectin test due to COVID-19 infection risk to the staff. Although home based calprotectin kits are also available, families using them have reported their use cumbersome and difficult to process tests at home. In addition, calprotectin results from other laboratories may be difficult to access. These limitations led to the development of a new regional service, in which samples taken at home are posted to the hub hospital laboratory (where the IBD clinic is based) for Calprotectin testing.

Aim To study the benefits of offering a service for posting faecal samples for calprotectin testing to a hub laboratory.

Methods Children (0-18 years) with IBD in the Wessex region, UK needing a calprotectin test were given postal faecal calprotectin packs (PFCP), either by hand in clinic or posted to their home. Each PFCP contained a labelled specimen bottle with immunology request form, bio-packaging box, sealable return bag (UN3373 compliant) with attached freepost label and instruction sheet. A Calprotectin cut off level of $<200$ was used as normal.

Results 63 patients $(M=34,54 \% \& F=29,46 \%)$ were given PFCP between 27th July \& 5th of Nov 2020 with $52.4 \%$ posted PFCP and $47.6 \%$ given PFCP by hand in the paediatric GI clinic. The patients resided at a mean distance of 41.6 miles (1 SD $=24.1$ miles) as the crow flies from the hospital. A mean of 25 days $(1 \mathrm{SD}=10$ days) were taken from posting/handing of PFCP to the lab test result being obtained.

The PFCP was returned by 50 patients (79.4\% compliance) with a diagnosis of Crohn's disease 34.9\%, UC 28.6\%, IBDU $7.9 \%$, oral ulcers $4.8 \%$ and $23.8 \%$ of patients referred for endoscopy with IBD like symptoms. $30 \%$ of the patients with IBD (15/50) posting the PFCP had an abnormal test result. This led to a change in management in $40 \%$ of the patients. In the patient group referred with suspected IBD only $1 / 15$ patients had an abnormal calprotectin test. $70 \%$ of patients with a normal test were able to be reassured without further investigation.

Conclusion This is the first reported series, offering to a large region a robust method for samples to be taken at home and posted to a central hub laboratory for calprotectin testing during the COVID-19 pandemic. Test results were readily available, being performed in the same hospital site as the IBD clinic. Compliance with the new PFCP remains high with $80 \%$ using the new PFCP service, with value in early identification of patients who may not have much in terms of symptoms and avoidance of endoscopy in others with a normal calprotectin.

\section{P48 $\quad$ SHOCK COLON}

${ }^{1}$ Milda Jancauskaite, ${ }^{2}$ Alison Campbell, ${ }^{2}$ Bruce Jaffray, ${ }^{1}$ Sean Marven. 'Department of Paediatric Surgery, Sheffield Children's Hospital; ${ }^{2}$ Department of Paediatric Surgery, Great North Children's Hospital, Newcastle

10.1136/flgastro-2021-bspghan.57

Introduction Non-occlusive mesenteric ischaemia (NOMI) is rare in children. There are individual case reports of ischaemic colitis, with various underlying causes for the acute deterioration. The likely mechanism is hypoperfusion/reperfusion injury. The outcome tends to be poor. Potentially; because the initial hit to the whole system is so significant, or possibly because of the toxicity of the colonic insult. Promptness of colonic resection does not seem to improve survival.

In our institutions we recognised a series of critically ill patients with a similar pattern of colonic injury. The patients had a comparable clinical picture and outcome. Therefore, we hypothesised; a similar underlying pathophysiology might be responsible.

By collaborating, the expectation was that we would identify key learning points.

Aim We aimed to identify patterns in presentation and correlate these with, surgical and pathology findings. By studying our cohort and reviewing the existing literature, we hoped to identify a possible means of improving survival.

Subject and Methods We reviewed clinical notes, histology, radiology and laboratory results of 4 consecutive cases of idiopathic colonic gangrene associated with acute cardiovascular collapse. Patients presented over a 2-year period to our 2 institutions (both providing tertiary paediatric surgery). We reviewed the literature on young adults and children with ischaemic colitis and non-occlusive mesenteric ischaemia. 